Service social

\title{
Analyse de l'articulation du système de prévention des violences sexuelles sur mineurs dans le canton de Vaud - Suisse
}

\section{Raphaël Depallens}

Volume 63, numéro 1, 2017

Interventions en matière d'agressions sexuelles

URI : https://id.erudit.org/iderudit/1040032ar

DOI : https://doi.org/10.7202/1040032ar

Aller au sommaire du numéro

Éditeur(s)

École de service social de l’Université Laval

ISSN

1708-1734 (numérique)

Découvrir la revue

Citer cet article

Depallens, R. (2017). Analyse de l'articulation du système de prévention des violences sexuelles sur mineurs dans le canton de Vaud - Suisse. Service social, 63(1), 73-85. https://doi.org/10.7202/1040032ar
Résumé de l'article

Ce travail vise à décrire la manière dont s'articule le programme de prévention des violences sexuelles du système scolaire vaudois à l'intention des enfants de 4 à 12 ans. L'intérêt de ce document repose sur la mise en relation d'aspects théoriques et de données empiriques permettant d'évaluer la réalité des pratiques en termes de détection précoce et d'intervention dans un contexte scolaire. Les données ont été récoltées par interviews semi-dirigées auprès d'un groupe de professionnelles de l'école (enseignantes, psychologue, infirmière scolaire...) de l'Unité de prévention et de promotion de la santé en milieu scolaire, ainsi qu'auprès de structures externes à l'État (association, experts, etc.). Il ressort de ce travail que le système de prévention actuellement en place est peu structuré et manque de moyens. 


\title{
Analyse de 1'articulation du système de prévention des violences sexuelles sur mineurs dans le canton de Vaud - Suisse
}

DEPALLENS, Raphaë1

\begin{abstract}
RÉSUMÉ
Ce travail vise à décrire la manière dont s'articule le programme de prévention des violences sexuelles du système scolaire vaudois à l'intention des enfants de 4 à 12 ans. L'intérêt de ce document repose sur la mise en relation d'aspects théoriques et de données empiriques permettant d'évaluer la réalité des pratiques en termes de détection précoce et d'intervention dans un contexte scolaire. Les données ont été récoltées par interviews semi-dirigées auprès d'un groupe de professionnelles de l'école (enseignantes, psychologue, infirmière scolaire...) de l'Unité de prévention et de promotion de la santé en milieu scolaire, ainsi qu'auprès de structures externes à l'État (association, experts, etc.). II ressort de ce travail que le système de prévention actuellement en place est peu structuré et manque de moyens.
\end{abstract}

Mots-clés : abus sexuel, maltraitance, éducation sexuelle, prévention, médecine sociale et préventive.

\begin{abstract}
This work aims to describe the way in which the program of prevention of the sexual violence in the school system of Vaud is organized for the children of 4 to 12 years. The interest of this document is based on the linking of theoretical aspects and empirical data making possible to evaluate the reality of practices in terms of early detection and intervention in a school context. The data were collected by semi-directed interviews with a group of school professionals (teachers, psychologists, school nurses, etc.) from the School Health Prevention and Promotion Unit and ith structures outside the State (association, experts, etc.). It emerged from this work that the prevention system currently in place is poorly structured and lacking in means.
\end{abstract}

Keywords: sexual abuse, maltreatment, sex education, prevention, social and preventive medicine.

\section{INTRODUCTION}

\section{Débutons par un nombre : 89184}

Peu de personnes savent qu'il s'agit d'une estimation du nombre de personnes (375 781 hommes / 391716 femmes en 2015 sur le canton de Vaud) ayant été victimes de violences sexuelles avec contact physique avant l'âge de 17 ans ( $8 \%$ des hommes et $22 \%$ des femmes [Averdijk et Eisner, 2014]). Trop souvent, les violences sexuelles sur mineurs sont considérées comme une exception et il n'est pas rare de voir que cette estimation provoque des réactions de stupéfaction. Cet article, repris d'un travail de bachelor, est donc issu d'une réflexion qui concerne le nombre de personnes victimes de violences sexuelles durant l'enfance. II s'intéresse aux moyens de prévention pensés et mis en œuvre qui visent à réduire le nombre élevé de jeunes victimes. De manière plus spécifique, cette recherche analyse le-s système-s de prévention des violences sexuelles dans le système scolaire vaudois auprès des enfants de 4 à 12 ans afin d'en évaluer la pertinence. 
Dans cette introduction, il semble évident de définir ce qui est appelé violence sexuelle dans ce document. Il s'agit « d'une dénudation, des attouchements ou des rapports sexuels entre personnes de maturité physique et psychique différente » (Dorais, 1997, p. 16), à partir de «trois et cinq ans » (Hébert, Cyr et Tourigny, 2011, p. 9) de différence d'âge. Cette transgression est «imposée par manipulation, abus de confiance, chantage, coercition menace ou violence » (Dorais, 1997, p. 16). II semble plus judicieux de parler de violence sexuelle et non pas d'abus sexuel, le terme « abus » diminue la reconnaissance, en termes de violence, de l'impact possible sur la personne victime, et se centre particulièrement sur l'acte commis par l'auteur (Krug et al., 2002, p.166).

Le contexte joue souvent un rôle majeur, je vais tenter d'en donner quelques éléments. Pour le contexte historique, en 1986, l'affaire du "Sadique de Romont » alias Michel Peiry (Salas, 2010) et 10 ans plus tard "l'affaire Dutroux » (Licata et Klein, 2000) auront sans aucun doute un impact décisif dans la volonté d'amener les questions de prévention de violences sexuelles à l'école et cela surtout chez les plus petits. De 1997 à 2000, l'affaire d'Outreau semble aussi avoir un impact indirect sur l'éducation sexuelle et plus spécifiquement sur la prévention des violences sexuelles. Toujours en 1986, la conférence d'Ottawa sur la promotion de la santé débouche sur la charte d'Ottawa, document fondateur de la promotion de la santé incluant les questions de santé sexuelle (Cortolezzis et Muheim, 2002, p. 68). En 1989, les Nations unies créent une Convention relative aux droits de l'enfant, ratifiée en 1997 par la Suisse. Cette convention donne alors une forme de statut particulier aux enfants, une reconnaissance juridique spécifique et des considérants en termes de besoins, de sécurité et de bien-traitance (UNICEF,1989). En 1990, le premier cours d'éducation sexuelle a lieu dans une classe enfantine avec une large place donnée aux violences sexuelles (Cortolezzis et Muheim, 2002, p.69). Les mouvements associatifs vaudois venant en aide aux enfants et adolescent-e-s victimes de violence sexuelle ainsi qu'à leur famille voient le jour dans la première moitié des années 1990 (les associations Faire le Pas et Familles solidaires, regroupées aujourd'hui en une seule entité, ESPAS, et l'association DIS NO). La mise sur pied de ces associations permet alors de problématiser de manière publique les questions de violence sexuelle. Une activation des pouvoirs publics en ce sens intensifie la prévention. Les révélations de 2002 sur des affaires de violences sexuelles en lien direct avec l'Église catholique, principalement à Boston, puis le dévoilement de nouveaux scandales en 2010 ont aussi contribué à nourrir le débat public et à interroger la place tenue par la prévention dans notre société et plus particulièrement dans la société suisse.

Une grande partie de la prévention des violences sexuelles sur mineurs se fait par le biais de l'éducation sexuelle (mandat externe à la Fondation Profa de 10 heures sur l'entièreté de la scolarité). À ce titre, l'initiative populaire fédérale «Protection contre la sexualisation à l'école maternelle et à l'école primaire » (Administration fédérale de la Conférédation suisse, 2016), déposée en décembre 2013, a été retirée en juin 2015, suite aux recommandations du parlement. II a été constaté que, selon bon nombre de répondant-e-s, elle a porté préjudice à la prévention et a possiblement ajouté un sentiment de légitimité à ceux et celles qui pensaient que les questions de sexualité sont l'affaire de la famille. Ce texte mentionnait qu'un " cours destiné à la prévention des abus sexuels envers les enfants peut être dispensé à partir de l'école maternelle » mais « ce cours n'aborde pas l'éducation sexuelle » (Administration fédérale de la Conférédation suisse, 2016).

II est aussi nécessaire de remarquer que certaines études actuelles vont dans le même sens en affirmant qu'« il n'est pas certain que [...] les programmes de prévention permettent de réduire la violence sexuelle envers les enfants » (Averdijk et Eisner, 2014, p. 118) et que " ces programmes peuvent également avoir certains effets préjudiciables » (Averdijk et Eisner, 2014, p. 118) comme des peurs ou une dépendance affective plus marquées (Averdijk et Eisner, 2014, p. 121). 
Finalement, les spécialistes du terrain ont le sentiment que la thématique - peu abordée au niveau politique et sociétal - constitue un sujet sensible et encore partiellement tabou.

Ces divers éléments montrent qu'au-delà de la sensibilité de la thématique et par certains aspects de sa gravité, elle s'inscrit dans un contexte plutôt en tension. Je tenterai de me centrer sur une vision préventive ainsi que sur le repérage précoce (intervention précoce), car ceux-ci sont porteurs d'utopie et d'espoir, dès lors que l'on souhaite tenter d'infléchir la courbe des violences sexuelles dans l'enfance.

\section{Méthodologie et entretiens}

La méthologie utilisée dans ce travail s'appuie sur plusieurs ouvrages, comme ceux de Singly, 2005; Liamputtong, 2011; Naud, Tremblay et Chicoine, 2008 ; Vaughn, Shay Schumm et Sinagub, 1996). Ces ouvrages m'ont permis d'utiliser des outils déjà pensés pour recueillir les données, à savoir le questionnaire, l'interview et le focus group.

Pour les questionnaires adressés à l'association ESPAS, au service d'éducation sexuelle de Profa, à l'Unité PSPS ainsi qu'à Madame Carol Gachet (experte en ICP), la préférence d'un entretien a été évoquée par chacun-e de ses répondant-e-s. J'ai donc rencontré :

Professionnelles du service d'éducation sexuelle de Profa, le 9 novembre 2015 (PROFA Fondation reconnue d'utilité publique par le Canton de Vaud - Offre des prestations en matière de santé sexuelle, de grossesse, de sexualité, de relations de couple, ou à ceux et celles qui ont subi des infractions d'ordre physique, sexuel ou psychique. 6 services : éducation sexuelle, consultation de santé sexuelle, planning familial, conseil en périnatalité, consultation de couple et de sexologie, centre LAVI).

Experte en Intervention de crise et prévention (ICP), le 25 novembre 2015 : Madame Carol Gachet, Directrice et fondatrice de I'ICP - Psychologue FSP - Fondatrice de l'association Faire Le Pas, ancienne coordinatrice de l'association Familles Solidaires - Initiatrice et responsable du projet GRAFIC - Experte pour l'Office fédéral des assurances sociales (OFAS).

Responsables de Unité PSPS (UPSPS ou Unité PSPS - unité de promotion de la santé et de prévention en milieu scolaire), le 6 janvier 2016.

Intervenant de l'association ESPAS (ESPAS - association - espace de soutien et de prévention abus sexuels), le 11 janvier 2016.

École primaire vaudoise, le 19 novembre 2015 : Focus Group composé d'une enseignante ordinaire (EO), d'une enseignante de soutien (ES), d'une psychologue scolaire (PS) et d'une infirmière scolaire (IS).

Doyenne d'établissement primaire, par questionnaire.

Questions, hypothèses et résultats

Question 1 : Comment le système de prévention des violences sexuelles est-il conçu, mis en place, réalisé et évalué dans le milieu scolaire public vaudois?

Hypothèse 1 : Le système de prévention des violences sexuelles sur mineurs semble s'articuler au travers d'une organisation qui manque de clarté et qui est très complexe. De plus, il semble qu'aucun modèle ou vision ne permette d'orienter une stratégie, de mettre en place une organisation. L'éducation sexuelle de Profa porte donc seule et avec de faibles moyens la responsabilité de cette thématique. Finalement, les impacts secondaires (peur, anxiété) que peuvent engendrer certains programmes jouent un rôle dans la manière dont la promotion de la prévention s'organise. 
Tentative de réponse 1 : La récolte de donnée tend à vérifier ce qui ressort de la partie théorique, à savoir la difficulté de faire émerger une articulation précise, une vision spécifique du système de prévention ainsi que des concepts communs et une stratégie clairement définie qui permettent de mettre celle-ci en œuvre. L'UPSPS semble détenir la vision la plus globale de ce qui se fait en la matière. Au contraire il apparaît difficile pour certain-e répondant-e d'identifier les ressources existantes. L'hypothèse qui reposait sur le fait que l'éducation sexuelle joue un rôle central dans le dispositif se vérifie. II s'avère que le temps à disposition de Profa semble être en quantité minime pour traiter le sujet, comme en témoignent les répondant-e-s. Le service d'éducation sexuelle semble avoir mis en place les recommandations du rapport de l'IUMSP, mais a été freiné par des demandes de budget supplémentaire qui ont été refusées. Le seul élément qui n'a pas été modifié suite à cette évaluation et qui figurait dans les recommandations concerne la mixité des cours d'éducation sexuelle alors même que tous les répondant-e-s reconnaissent l'intérêt de séparer momentanément les deux sexes afin d'aborder le sujet.

Certain-e-s répondant-e-s évoquent le fait que l'école n'a peut-être pas de rôle central à jouer dans une problématique privée qui concerne le cadre familial. Cette idée se retrouve en opposition complète avec celle d'autres répondant-e-s qui évoquent l'importance de l'école en termes de prévention et qui font de cette question un problème public qui doit être géré en grande partie par l'État. Comme l'a évoqué ESPAS, l'école doit être bien-traitante et offrir un espace de prévention permettant d'aborder diverses thématiques générales de prévention. Les réponses données par le focus group nous permettent de constater que la formation sur le sujet ne répond pas aux besoins des professionel-le-s de l'école. Ces professionnel-le-s du milieu scolaire disent se sentir hors du système de prévention. Le fait que le focus group sous-représente le nombre de personnes victimes de violence sexuelle tend aussi à révéler le fait que la problématique est mal connue. Dans ce sens, le projet SESAME pourrait amener des informations utiles pour thématiser la problématique de la prévention au sein des établissements scolaires. II s'agirait également de fournir des ressources de proximité selon la nature du projet susceptible d'être mis sur pied.

En ce qui concerne les effets secondaires possibles associés aux programmes de prévention, il paraît aberrant de penser que les programmes actuels, lorsqu'ils sont fondés sur des connaissances reconnues et des moyens importants, puissent générer de la peur ou de l'angoisse. Par ailleurs, comme l'évoque ESPAS, la peur peut aussi avoir une utilité. Elle n'est pas forcément dramatique et peut avoir une fonction du moment qu'elle n'est pas traumatique. ESPAS relève que certains enfants ont aussi des angoisses concernant des tests de vocabulaires en allemand. Ce n'est pas pour autant qu'on arrête d'enseigner cette branche.

Pour conclure, il semble donc qu'hormis le fait que certain-e-s répondant-e-s associent des effets secondaires à certains programmes de prévention, les hypothèses émises suite à la partie théorique se vérifient.

Question 2: Quels sont les moyens mis en place pour réaliser le système de prévention des violences sexuelles sur mineurs?

Hypothèse 2 : Les moyens mis à disposition pour la détection précoce et l'intervention semblent suffisants. En ce qui concerne la prévention, les moyens paraissent insuffisants en termes de temps, d'offre et de financement.

Tentative de réponse 2: L'hypothèse émise se vérifie du fait que tous les répondant-e-s reconnaissent, d'une manière ou d'une autre, la nécessité de fournir plus de moyens de prévention. Chacun-e développe toutefois des arguments différents pour justifier ce manque de moyen. Comme dans la partie théorique, aucun-e répondant-e n'est en mesure de chiffrer le coût de la prévention et 
il est possible que certain-e-s n'aient sciemment pas souhaité évoquer le sujet. II est nécessaire de préciser qu'en ce qui concerne la détection précoce et la prise en charge des personnes victimes, le dispositif est reconnu comme fiable et de qualité.

II ressort que les 10 heures d'éducation sexuelle sont insuffisantes pour aborder le sujet de manière globale. La position de Profa, sollicitée par un mandat externe, peut éventuellement expliquer le faible financement des services demandés. Une autre situation serait envisageable si Profa appartenait à l'État. En ce sens, le cahier des charges de Profa est beaucoup trop conséquent par rapport au financement alloué

Le manque de connaissances sur le sujet, l'absence d'un débat public et politique peuvent être considérés comme des freins à l'obtention de financements conséquents accordés par les politiques. Dans le canton de Vaud, la prévention sur une thématique se fait par vague, lorsqu'un sujet est médiatisé. Celui-ci est vite remplacé lorsqu'une nouvelle thématique est mise sur le devant de la scène médiatique. II semble alors difficile de mettre en place un système de prévention efficace, car fondé sur une connaissance globale des enjeux de prévention et construit sur le long terme.

Cependant, nous possédons trop peu d'éléments concrets pour répondre à cette question. Nous pouvons seulement constater que les moyens mis en œuvre sont insuffisants et/ou souvent mal utilisés.

Question 3 : À qui s'adresse la prévention des violences sexuelles sur mineurs ?

Hypothèses 3 : La prévention est axée uniquement sur les potentielles victimes et sur les possibilités d'intervention auprès des personnes victimes.

Tentative de réponses: L'hypothèse se vérifie pleinement: la prévention ne s'adresse qu'aux victimes potentielles de violence sexuelle. Les victimes continuent alors à porter le double poids de ne pas avoir su se protéger conformément aux indications de la prévention données tout en subissant les impacts d'être victime. La littérature sur le sujet et tous les répondant-e-s ont confirmé l'intérêt d'inclure les parents, les enseignant-e-s ainsi que l'importance de s'adresser aux auteur-e$s$ ayant ou étant susceptibles de passé à l'acte. Certain-e-s répondant-e-s sont même allé-e-s plus loin en parlant de l'intérêt de considérer la question de la prévention de manière plus systémique.

Les violences sexuelles sur mineurs semblent encore générer une forme de tabou, comme cela a pu apparaître dans les entretiens. La prévention des violences sexuelles sur mineurs est de fait confrontée à ces tabous qui font des violences sexuelles un sujet de société malheureusement peu débattu et souvent même passé sous silence. Le Canada constitue un exemple intéressant du fait qu'il semble avoir laissé de côté le tabou pour mettre en place une démarche préventive de sensibilisation globale.

En ce qui concerne la politique, il faut relever que la problématique des violences sexuelles ne semble pas traitée de manière prioritaire. Au niveau fédéral, mais aussi par le biais de l'OMS, les recommandations et publications sur le sujet font toutefois ressortir des éléments importants qui mettent en lumière une réelle problématique. Ces documents proposent aussi des informations et des outils pour la mise en place de systèmes de prévention coordonnés. En ce sens, on pourrait penser que la mise en place d'une politique en la matière devrait être facilitée. Par ailleurs, on peut constater qu'aucune structure fédérale n'est engagée de manière pérenne et interne sur le sujet. Comme dans le canton de Vaud, l'engagement se fait à l'extérieur de l'État avec des moyens jugés relativement faibles. II est nécessaire de rappeler que le Canton de Vaud n'a pas jugé nécessaire de mettre en place le programme national proposé par la Fondation pour la protection de l'enfance 
"Mon corps est à moi !", jugé trop conséquent. II faut relever que les expert-e-s de l'OFAS ont jugé ce programme comme pertinent. Dans la vision d'une stratégie politique, l'organisation (qui gère quoi et comment ? qui coordonne l'ensemble ?) et l'articulation (quelles relations entre les différents organismes et acteurs-trices ?) du système de prévention semble manquer de coordination, de moyens financiers et de lisibilité. Par ailleurs, il paraît spécialement clair que l'unité PSPS constitue la référence de l'État en la matière et le service de l'éducation sexuelle le prestataire de service pour la mise en place sur le terrain de la prévention des violences sexuelles sur mineurs.

Pour le volet financier, hormis ce qui concerne les prestations de Profa financées par l'Unité PSPS et le SSP, il est très difficile de pouvoir chiffrer précisément l'investissement financier. En ce sens il serait aussi intéressant de chiffrer, par le biais d'étude, le coût direct (soins, justice, prise en charge de l'auteur-e-s et de la victime...) et indirect (manque à gagner, impact sur la vie, impact sur l'entourage et perte de productivité...) des violences sexuelles sur mineurs pour les comparer aux investissements financiers en prévention.

Considéré comme le moyen unique et reconnu de prévention des violences sexuelles, le rôle central du service d'éducation sexuelle de Profa semble prouvé. Or il serait également intéressant de favoriser une prévention plus globale axée sur le vivre ensemble, le respect de soi et de l'autre, le consentement. Ce type de prévention plus globale, que l'on retrouve dans la théorie, a été plébiscité par l'unité PSPS, l'éducation sexuelle, Mme Gachet et ESPAS.

Le paradoxe qui réside dans le mandat donné à Profa est le fait que l'éducation sexuelle ne bénéficie que de 10 périodes durant la scolarité pour évoquer de nombreux sujets (contraception, VIH et autres IST, genre, orientation sexuelle, sexualité...). II semble que les moyens fournis à Profa pour réaliser son mandat sont bien en deçà de ce qui serait nécessaire pour réaliser une prévention optimale. Par ailleurs, l'intervention de l'éducation sexuelle offre aux jeunes des prestations adaptées de qualité. Les outils sur lesquels se reposent les éducateurs-trices en santé sexuelle de Profa sont fiables et actuels. D'autre part, en ce qui concerne la mixité des cours d'éducation sexuelle quand des questions de violences sexuelles sont évoquées, il semblerait nécessaire de pouvoir séparer filles et garçons.

Les cours d'éducation sexuelle ne sont pas obligatoires, mais sont suivis par quasi tous les élèves. Cependant, il est intéressant de constater que c'est le seul cours facultatif du cursus scolaire, puisque les parents peuvent effectivement refuser que leur-s enfant-s y participent.

Le rôle que l'école peut jouer dans la prévention des violences sexuelles sur mineurs semble diviser. Une partie des répondant-e-s pense que la problématique est externe à l'école. De ce fait, l'école peut difficilement agir dans un contexte où elle ne serait pas légitime. La question des violences sexuelles sur mineurs se situe dans trois sphères à savoir : la sphère de l'enfance, la sphère de la violence et la sphère de la sexualité. La sphère de la sexualité étant considérée comme faisant partie de l'intime, faisant donc partie du domaine privé, l'école semble ne pas savoir comment aborder cette thématique afin de ne pas intervenir dans le cadre du privé. Les théories sur ce sujet ne paraissent pas apporter de réponses claires, ce qui maintient un certain flou. En ce sens, la fonction de l'école consiste à favoriser les apprentissages scolaires ainsi qu'une action de santé communautaire sans constituer la figure de proue de la prévention. L'autre partie des répondant-es semble dire que la prévention fait partie intégrante des apprentissages appartenant au vivreensemble. II est également reconnu que le mandat de l'école vise à réduire les inégalités et à favoriser le bon développement de l'enfant. Cette partie des répondant-e-s constate que l'école a encore beaucoup de progrès à faire en ce sens et manque cruellement de moyens. On peut donc constater qu'il n'y a pas consensus sur le sujet ni d'ailleurs de discussions sur ces opinions divergentes. 
En termes de formation des enseigant-e-s, on peut constater un manque certain. Que cela soit en termes de formation initiale ou continue, il appert que l'infime part donnée à la prévention et à la détection précoce ne semble pas permettre aux enseignant-e-s d'avoir des outils suffisants pour se sentir légitimes. II semble que le projet SESAME pourrait apporter un relais et des connaissances importantes au sein de l'école sur ce sujet. Toutefois en ce qui concerne la prévention, peu de mesures sont prises pour offrir des ressources et mettre en place une prévention optimale.

Pour conclure, la faiblesse du système actuel de prévention se révèle au travers de nos trois réponses. Cette faiblesse s'expliquerait par le manque de volonté des politiques qui ne souhaitent pas considérer pleinement la question de la prévention, par le côté tabou que la sexualité tient dans notre société et par les moyens très modestes qui sont actuellement alloués à une problématique de grande ampleur.

\section{RECOMMANDATIONS}

Dans cette partie vous trouvez par thèmes des propositions de recommandations qui sont issues de la concertation entre la partie théorique et les données récoltées. Ces recommandations offrent aussi une réflexion au-delà de ce modeste travail.

\section{Système et articulation de la prévention}

Proposer un modèle holistique (Bronfenbrenner), qui comprend les auteur-e-s de violences sexuelles sur mineurs ainsi que tous les acteurs-trices (rôle de chacun-e dans chaque niveau).

Centraliser la prévention dans ce domaine autour d'un comité de pilotage.

Attribuer un mandat de coordination de la prévention.

Établir un plan de prévention comprenant les moments de l'intervention (prévention - détection précoce - intervention) et les niveaux d'intervention.

Établir un état des lieux et un listing des structures concernées (élaborer une brochure informative).

Documenter l'impact social et les coûts des violences sexuelles durant l'enfance.

Offrir une prévention positive (non basée sur la peur ou l'anxiété).

\section{Politique et société}

Tendre à créer une politique préventive en la matière.

Allouer des moyens financiers adéquats et lisibles (système politique et financier moins fastidieux [2 départements + Profa + école + association...]).

Créer des campagnes de sensibilisation du grand public (prise de conscience).

\section{L'éducation sexuelle}

Obtenir des moyens financiers en nombre d'heures allouées en adéquation avec le mandat.

Insérer ce service dans l'articulation d'un système de prévention.

Proposer d'aborder le sujet dans des groupes non mixtes.

Exporter l'expertise de l'éducation sexuelle de Profa dans les établissements scolaires. 


\section{L'école}

Offrir à la plupart des enfants du canton de Vaud une prévention optimale afin de favoriser les facteurs de protection et diminuer les facteurs de risque.

Concevoir la prévention à l'école comme une branche à part entière pouvant traiter de sujets multiples mais ayant une base commune.

Disposer de document favorisant la communication sur le sujet et l'emploi de concepts et d'une terminologie communs.

Former les professionnel-le-s de l'école et de l'éducation, voire des soins (HEP, EESP, HESAV, Université de médecine...), à la prévention, la détection précoce et l'intervention.

\section{Conclusion}

Le système de prévention des violences sexuelles semble reposer principalement sur les 10 périodes octroyées au service d'éducation sexuelle de Profa durant la scolarité obligatoire. À la demande des établissements scolaires, l'unité PSPS fait aussi de la prévention, par exemple sur la problématique du climat scolaire ou de la violence. La conception du système de prévention ne semble pas découler d'une politique en la matière. En ce sens, l'organisation et la coordination des multiples institutions traitant partiellement ou totalement de prévention des violences sexuelles sur mineurs ne bénéficient pas d'une vision et d'un modèle communs. La seule structure ayant été au bénéfice d'une évaluation est le service d'éducation sexuelle de Profa (rapport de I'IUMSP). II semblerait qu'aucune autre évaluation publique n'ait été faite dans le canton de Vaud au sujet de la prévention. Les moyens financiers sont alloués à Profa par l'État via l'unité PSPS (SESAF) et le SSP. Les autres moyens recensés sont alloués à des organismes n'ayant pas accès à l'école. Les moyens financiers restent difficilement quantifiables à cause de l'éparpillement des différents organismes et d'un système de prévention dilué. Les ressources visant à financer des campagnes de prévention, mais aussi des brochures et autres outils favorisant la prévention semblent maigres. Concernant l'intervention ou la détection précoce, il semble que notre système soit doté d'une solide prise en charge ainsi que de moyens financiers adaptés encadrés par une législation et une politique claires en la matière.

II appert que le système de prévention des violences sexuelles est minimum et peu satisfaisant au vu des éléments détenus dans ce document. Le manque de vision et de stratégie en la matière, de moyens financiers, de connaissances et de formations des professionnel-le-s de l'école et finalement de volonté politique sur le sujet tendent à maintenir un système de prévention pauvre. En revanche, en ce qui concerne la prise en charge des victimes mais aussi celle des auteur-e-s de violences sexuelles (détection précoce et intervention), les moyens sont jugés comme étant bons et de qualité.

Raphaël Depallens

Éducateur social (Bachelor en éducation social HETS\&SA - Lausanne - Suisse) Fondateur et responsable du Pôle Agression et Violence (PAV)

\section{RÉFÉRENCES}

Absil, G., C. Vandoorne et M. Demarteau (2012). Bronfenbrenner, écologie du développement humain. Réflexion et action pour la promotion de la santé, Liège, École de santé publique. 
Administration fédérale de la Confération suisse (2016). Initiative populaire fédérale "Protection contre la sexualisation à l'école maternelle et à l'école primaire ». Récupéré de $<$ https://www.admin.ch/ch/f/pore/vi/vis432.html>.

Administration fédérale de la Confération Suisse (2016). Initiative populaire fédérale "pour l'imprescriptibilité des actes de pornographie enfantine". Récupéré de <https://www.admin.ch/ch/f/pore/vi/vis329.html>.

Ain, J. (dir.) (2010). Résiliences, Paris, Éditions ERES.

Alter-Reid, K., M.S. Gibbs, J.R. Lachenmeyer, J. Sigal et N.A, Massoth (1986). « Sexual abuse of children: A review of the empirical findings ", Clinical Psychology Review, vol. 6, n 4, p. 249-266.

Alami, S., D. Desjeux et I. Garabuau-Moussaoui (2009). Les méthodes qualitatives, Paris, Presses universitaires de France.

Ancona, L., et F. Boillat (2012). Abus sexuels envers les enfants : éviter le premier passage à l'acte. État des lieux et analyse de la situation au niveau international. Perspectives pour la Suisse romande, Monthey, Éditions DIS NO.

Archambault, M., P. Martinet et O. Duperrex (2011). Promotion de la santé et prévention en milieu scolaire. Rapport intermédiaire de législature 2007-2012. Récupéré de <http://www.vd.ch/fileadmin/user_upload/organisation/dfj/sesaf/odes/Documentation/ODESRapport-legislature-2007-12.pdf>.

Association ESPAS (2016). Antenne Vaud. Récupéré de $<$ http://www.espas.info/espas/organisation/antenne-vaud/>.

Association DIS NO (2016). DIS NO en bref. Récupéré de <http://www.disno.ch/disno/>.

Association MIRA (2016). Service de prévention MIRA. Récupéré de $<$ http://www.vaudfamille.ch/N163574/service-de-prevention-mira.html>.

AVASAD (2016). Service santé scolaire. Récupéré de <http://www.avasad.ch/jcms/m_7018/fr/service-sante-scolaire>.

Averdijk, M., et M. Eisner (2014). Prévention de la violence. État actuel du savoir sur l'efficacité des approches ( $2^{\mathrm{e}}$ éd), Berne, Office fédéral des assurances sociales.

Blanchet, A., et A. Gotman (2015). L'entretien, Paris, Armand Colin.

Canton de Vaud (2016a). Autorités. Récupéré de <http://www.vd.ch/autorites/>.

Canton de Vaud (2016b). Départements. Récupéré de <http://www.vd.ch/autorites/departements/>.

Canton de Vaud (2016c). Thème: Incivilités, violence. Récupéré de <http://www.vd.ch/themes/formation/sante-a-lecole/projets-psps/incivilites-violence/>.

Canton de Vaud (2016d). Les Équipes PSPS. Récupéré de $<$ http://www.vd.ch/themes/formation/sante-a-lecole/equipes-psps/>. 
Caplan, G. (1964). Principles of Prevention Psychiatry, Oxford, Basic Books.

Centre national de ressources textuelles et lexicales (2012). «Programme ». Récupéré de $<\mathrm{http}: / /$ www.cnrtl.fr/definition/programme>.

Conseil de l'Europe (2007). Convention du Conseil de l'Europe sur la protection des enfants contre l'exploitation et les abus sexuels. Récupéré de <https://www.admin.ch/opc/fr/classifiedcompilation/20121286/index.html>. Le 27 septembre 2013, la Suisse a signé la Convention du Conseil de l'Europe sur la protection des enfants contre l'exploitation et les abus sexuels (entrée en vigueur en juillet 2014).

Conseil fédéral (2011). Loi fédérale sur l'encouragement des activités extrascolaires des enfants et des jeunes (Loi sur l'encouragement de l'enfance et de la jeunesse, LEEJ) du 30 septembre 2011. RS: 446.1. Récupéré de <https://www.admin.ch/opc/fr/classifiedcompilation/20092618/index.html>.

Cortolezzis, C., et D. Muheim (2002). L'éducation sexuelle dans le canton de Vaud (1969-2001). Un exemple d'intégration des risques dans une vision positive de la sexualité, Lausanne, Éditions Raisons de santé.

CHUV (2016). Centre de consultation Les Boréales. Récupéré de $<$ http://www.chuv.ch/psychiatrie/fiches-psychiatrie_details.htm?fiche_id=318>.

DGEO (Direction générale de l'enseignement obligatoire) (2016). Scolarité obligatoire. Récupéré de <http://www.vd.ch/themes/formation/scolarite-obligatoire/cursus-scolaire/>.

DGEO (Direction générale de l'enseignement obligatoire) (2012). Les indicateurs de l'enseignement obligatoire. Année scolaire 2011-2012. Récupéré de <http://www.vd.ch/fileadmin/user_upload/organisation/dfj/dgeo/fichiers_pdf/ indic_oct12_final.pdf>.

DFJC (Département de la formation, de la jeunesse et de la culture) (2001). Principes de collaboration et de communication dans l'intervention en faveur de mineurs en difficulté ou en danger dans leur développement, Lausanne, Canton de Vaud.

Dorais, M. (1997). Ça arrive aussi aux garçons. L’abus sexuel au masculin, Montréal, VLB éditeur.

Fabian, C., N. Käser, T. Klöti et N. Bachmann (2014). Critères de bonnes pratiques. Prévention de la violence juvénile dans la famille, à l'école et dans l'espace social, Berne, Office fédéral des assurances sociales.

Gordon R. (1983). "An operational classification of disease prevention », Public Health Reports, vol. $98, n^{\circ} 2$, p. 107-109.

Grand Conseil du Canton de Vaud (2004). Loi sur la protection des mineurs (LProMin) du 4 mai 2004. RS: 850.41. Récupéré de <http://www.rsv.vd.ch/rsvsite/rsv_site/doc.pdf?docld=5890\&Pvigueur=\&Padoption=\&Pcurrent_ver sion=9999\&PetatDoc=vigueur\&Pversion=\&docType=loi\&page_format=A4_3\&isRSV=true\&isSJL $=$ true \&outformat $=$ pdf\&isModifiante $=$ false $>$. 
Grand Conseil du Canton de Vaud (2011). Loi sur l'enseignement obligatoire (LEO) du 7 juin 2011. RS: 400.02. Récupéré de <http://www.rsv.vd.ch/direcocoon/rsv_site/doc.pdf?docld=870673\&Pvigueur=\&Padoption=\&Pcurrent_version=0\&PetatDoc $=$ vigueur\&Pversion=\&docType=loi\&page_format=A4_3\&isRSV=true\&isSJL=true\&outformat=pdf\&is Modifiante $=$ false $>$.

Grand Conseil du Canton de Vaud (2012). Loi d'application du droit fédéral de la protection de l'adulte et de l'enfant (LVPAE) du 29 mai 2012. RS: 211.255. Récupéré de <http://www.rsv.vd.ch/rsvsite/rsv_site/doc.pdf?docld=944443\&Pvigueur=\&Padoption=\&Pcurrent version $=9999 \& P e t a t D o c=v i g u e u r \& P v e r s i o n=\& d o c T y p e=l o i \& p a g e \_f o r m a t=A 4 \_3 \& i s R S V=t r u e \& i s S$ $\mathrm{JL}=$ true \&outformat=pdf\&isModifiante=false $>$.

Guide social romand (2016). Mauvais traitement à l'encontre des mineurs. Récupéré de $<$ http://www.guidesocial.ch/fr/fiche/989/>.

Halpérin, D.S., P. Bouvier, P.D. Jaffé, R.L. Mounoud, C.H. Pawlak, C. H., J. Laederach... et F. Astié (1996). " Prevalence of child sexual abuse among adolescents in Geneva: results of a cross sectional survey », BMJ, vol. 312, n 7042, p. 1326-1329.

Hébert, M., M. Cyr et M. Tourigny (dir.) (2011). L'agression sexuelle envers les enfants, Québec, Presses de l'Université du Québec.

HEP-Vaud (Haute École pédagogique) (2011a). Référentiel de compétences professionnelles. Récupéré de <https://www.hepl.ch/cms/accueil/formation/referentiel-de-competences.html>.

HEP-Vaud (Haute École pédagogique) (2011b). Promotion de la santé et prévention en milieu scolaire. Récupéré de <https://www.hepl.ch/cms/accueil/formation/unites-enseignement-etrecherche/developpement-enfant-a-adulte/thematiques-transversales/promotion-santeprevention.html>.

Jeunesetviolence.ch (2015). Programme de prévention de la Confédération, des cantons, villes et communes. Récupéré de <http://www.jeunesetviolence.ch/fr.html>.

Koutaissoff, D., F. Ischy, B. So-Barazetti, G. Meystre-Agustoni et F. Dubois-Arber (2009). Rapport d'évaluation des prestations du service d'éducation sexuelle de Profa en milieu scolaire, Lausanne, Institut universitaire de médecine sociale et préventive.

Krug, E.G., L.L. Dahlberg, J.A. Mercy, A. Zwi et R. Lozano-Ascencio (dir.) (2002). Rapport mondial sur la violence et la santé, Genève, Organisation mondiale de la santé.

Larousse (2016). « Vidéotex ». Récupéré de $<$ <ttp://www.larousse.fr/dictionnaires/francais/vidéotex/81897>.

Liamputtong, P. (2011). Focus group methodology. Principles and Practice, Los Angeles (CA), Sage Publication, Inc.

Licata, L., et O. Klein (2000). "Situation de crise, explications profanes et citoyenneté : l'affaire Dutroux », Cahiers Internationaux de Psychologie Sociale, nos $47-48$. 
Ministère de l'Éducation nationale (2002). Prévention et traitement des violences sexuelles. Récupéré de

$<$ http://media.eduscol.education.fr/file/Action_sanitaire_et_sociale/52/8/guide_prevention_violenc es_sexuelles_144528.pdf>.

Naud, D., R. Tremblay et $\mathrm{H}$. Chicoine (2008). « Carte méthodologique d'un groupe témoin », Cahiers de géographie du Québec, vol. 52, n 145, p. 81- 94.

OMS et ISPCAN (Organisation mondiale de la santé et International Society for Prevention of Child Abuse and Neglect) (2006). Guide sur la prévention de la maltraitance des enfants : intervenir et produire des données, Genève, Organisation mondiale de la santé et International Society for Prevention of Child Abuse and Neglect.

OMS (Organisation mondiale de la santé) (2013). Standards pour l'éducation sexuelle en Europe. Récupéré de <https://www.sante-sexuelle.ch/wp-content/uploads/2013/11/StandardsOMS_fr.pdf $>$.

PER (Plan d'études romand) (2012). Commentaires généraux pour la Formation générale. Récupéré de <https://www.plandetudes.ch/web/guest/fg/cg3>.

Profa (2016). Bases légales. Récupéré de <https://www.profa.ch/fr/fondation/bases-legales-0-7>.

Protection de l'enfance Suisse (2014). Simplification des droits d'aviser et application appropriées des obligations d'aviser, Berne, Protection de l'enfance Suisse.

Protection de l'enfance Suisse (2016). Parcours-découverte "Mon corps est à moi !". Récupéré de $<$ https://www.kinderschutz.ch/fr/parcours-decouverte-mon-corps-mappartient.html>.

Sanderson, C. (2004). The Seduction of Children: Empowering Parents and Teachers to Protect Children from Child Sexual Abuse, Londres et New York, Jessica Kingsley Publishers.

Salas, D. (2010). «Opinion publique et justice pénale. Une rencontre impossible ? », Le Temps des médias, vol. 15, $\mathrm{n}^{\circ}$ 2, p. 99-110

Schmid, C., M. Averdijk et M. Eisner (2012). Violences sexuelles envers des enfants et des jeunes en Suisse. Formes, ampleur, et circonstances du phénomène, Zurich, UBS Optimus Foundation.

Sethi, D., M. Bellis, K. Hughes, R. Gilbert, F. Miris et G. Galea (dir.) (2013). Rapport européen sur la prévention de la maltraitance des enfants, Genève, Organisation mondiale de la santé.

Singly, F. de (2005). Le questionnaire, Paris, Armand Colin.

South Estearn CASA (Centre Against Sexual Assault \& Family Violence) (2016). Récupéré de $<$ http://www.secasa.com.au/pages/theories-on-why-sexual-abuse-happens/the-four-preconditions-model/conditions-model/>.

SPJ (Service de protection de la jeunesse) (2015). Missions et organisation. Récupéré de $<$ http://www.vd.ch/fileadmin/user_upload/themes/sante_social/protection_jeunesse/Brochure_SP J_2015.pdf>. 
SPJ (Service de protection de la jeunesse) (2016). Mineurs en danger dans leur développement. Récupéré de <http://www.vd.ch/themes/vie-privee/enfance-et-jeunesse/protection-des-mineurs/>.

Spratt, T., J. Nett, L. Bromfield, J. Hietamäki, H. Kindler et L. Ponnert (2012). Child Protection Systems: An international comparison of "good practice examples" of five countries (Australia, Germany, Finland, Sweden, United Kingdom) with recommendations for Switzerland, Lund, The Swiss Project Fund for Child Protection.

UNICEF (Fonds des Nations unies pour l'enfance) (1989). Convention relative aux droits de l'enfant. Récupéré de <https://www.unicef.ch/fr/nous-aidons-ainsi/droits-de-lenfant/conventionrelative-aux-droits-de-lenfant>.

Vaughn, S., J. Shay Schumm et J. Sinagub (1996). Focus group interviews in education and psychology, Thousand Oaks (CA), Sage Publications, Inc. 